\title{
PRE-TEXTOS PARA LA TEORÍA DE LA JUSTICIA RAWLSIANA
}

\author{
EDWARD JAVIER ORDÓÑEZ \\ Universidad Santiago de Cali \\ ARÍSTIDES OBANDO CABEZAS \\ Universidad del Cauca \\ ARTURO HERNÁN ARENAS FERNÁNDEZ \\ Universidad Santiago de Cali
}

(c) (i) 


\title{
PRE-TEXTOS PARA LA TEORÍA DE LA JUSTICIA RAWLSIANA*
}

Resumen: El objetivo de establecer los conceptos iniciales o las primeras formas antes que fueran situados sustantivamente en la obra cumbre de John Rawls, Una teoría de justicia, fue la idea central de este informe. Se revisaron críticamente los documentos contractuales rawlsianos en los que se establecieron tales conceptos; estos son "Justice as Fairness" (1958), "The Sense of Justice" (1963) y "Distributive Justice" (1973). En ellos se encontraron las ideas germinales tales como la desestimación del criterio de utilidad, la primera formulación de los principios de justicia, la problemática del sujeto moral y su obligación con el cumplimiento con la normativa dada por los principios de justicia y, finalmente, el velo de ignorancia. Mismos que quizá hubieran desestimado algunas críticas hechas a Rawls.

Palabras clave: Rawls, teoría de la justicia, principios de justicia, sujeto moral.

\section{PRE-TEXTS FOR THE RAWLSIAN THEORT OF JUSTICE}

\begin{abstract}
The objective was established the first concepts or the first forms before they were located substantially in John Rawls's masterpiece: A Theory of Justice. It was the central idea in this dossier. The contractual rawlsians documents were reviewed critically and were found such concepts: "Justice as Fairness" (1958), "The Sense of Justice" (1963) y "Distributive Justice" (1973). These germinal ideas were found such as the rejection of the criterion of utility, the fist formulation of the principles of justice, the problematic of moral subject and its obligation with compliance with regulations given by the principles of justice, and finally the veil of ignorance. Perhaps themselves have rejected some criticisms of Rawls.
\end{abstract}

Keywords: Rawls, Theory of justice, Principles of Justice, Moral Subject.

Fecha de recepción: Diciembre 3 de 2015

Fecha de aceptación: Mayo 25 de 2016

Forma de citar (APA): Ordóñez, E.; Obando, A. y Arenas, A. (2016). Pre-textos para la teoría de la justicia rawlsiana. Revista Filosofía UIS, 15 (1), 47-60, doi: http://dx.doi.org/10.18273/revfil.v15n1-2016002

Forma de citar (Harvard): Ordóñez, E.; Obando, A. y Arenas, A. (2016). Pre-textos para la teoría de la justicia rawlsiana. Revista Filosofía UIS, 15 (1), 47-60.

Edward Javier Ordóñez: colombiano. Magíster en Filosofía por la Benemérita Universidad Autónoma de Puebla (México). Docente de Tiempo Completo en el Departamento de Humanidades y Artes de la Universidad Santiago de Cali (USC).

Correo electrónico: edward.javier11@gmail.com

Arístides Obando Cabezas: colombiano. Doctor en filosofía contemporánea, Doctor en derecho y Doctor en derecho y globalización, de la Universidad Autónoma del Estado de Morelos, México. Profesor asociado de la Facultad de Derecho en la Universidad del Cauca. Director del Grupo de Investigación Ética, Filosofía Política y Jurídica, de la Universidad del Cauca.

Correo electrónico: aristides.o@gmail.com

Arturo Hernán Arenas Fernández: colombiano. Candidato a Doctor en Comunicación; Magíster en administración de empresas; Comunicador social y periodista. Vicerrector Universidad Santiago de Cali.

Correo electrónico: arturoarenas@hotmail.com

*Artículo de reflexión.

Revista Filosofía UIS, Vol. 15, No. 1, enero-junio de 2016 pp. 47-60 


\section{PRE-TEXTOS PARA LA TEORÍA DE LA JUSTICIA RAWLSIANA'}

\section{Introducción}

El reto fue asumir filosóficamente la recepción latinoamericana de la obra de John Rawls, más precisamente la teoría de la justicia recepcionada en el contexto latinoamericano; fue un estudio filosófico comparativo que, desde la historia de las ideas de Horacio Cerutti, asume las reflexiones, problemáticas y debates sobre la justicia propuesta por el norteamericano. México y Colombia son los lugares de enunciación que permitieron esta comparación; Jesús Rodríguez Zepeda y Dora Elvira González, por México, y Óscar Mejía Quintana y Delfín Ignacio Grueso, por Colombia, fueron los autores elegidos. La elección de estos lugares fue fortuita, las facilidades al proceso de indagación fueron notorias; los autores en cambio no, ellos sí destacan trabajos rigurosos y profundos sobre la justicia como equidad y en la lista de futuros trabajos se quedaron algunos. Sin embargo, el trabajo de investigación exploró las discusiones de cinco autores colombianos que fueron los primeros en trabajar casi al tiempo la obra Una teoría de la justicia²; trabajo que precede y justifica la escogencia de los dos autores colombianos. Lo mismo se hizo con cinco autores mexicanos. La intención no fue otra que tomar a Rawls como piedra angular del trabajo.

${ }^{1}$ Este artículo es producto de la estancia de investigación en la Universidad Michoacana de San Nicolás de Hidalgo, México, para la realización del proyecto "Límites y retos de la recepción latinoamericana de la justicia rawlsiana: los casos Colombia- México"; con la asesoría del doctor Mario Teodoro Ramírez Cobián (a quien agradecemos infinitamente) y que hace parte de la iniciativa USC para la paz y el posconflicto. Proyecto y estancia financiados por la Universidad Santiago de Cali; código de radiación ante el consejo central de investigación: DGI-COCEIN no 568-621114-004.

${ }^{2}$ En adelante la llamaremos Una teoría. 
La obra Una teoría de Rawls revitalizó el quehacer de la filosofía política; este fue un punto de partida que justificó el porqué de la elección de este filósofo de Harvard. Rawls ganó un espacio significativo como teórico político gracias a la erosión de los elementos críticos de la teoría política por las guerras mundiales ${ }^{3}$ y a las discusiones estériles por la naturaleza de la filosofía, sus fines disciplinares y los filósofos/as políticos/as, esto es, la reflexión por la naturaleza de la disciplina indica la no concordancia entre los fines de la filosofía política, que se tornan prácticos, y el espíritu teorético del paradigma filosófico, y el cómo quienes la ejercen fueron (son y serán) sometidos a la evaluación desde las ciencias duras. Con Una teoría los especialistas posicionaron a Rawls con especial estima, desde sus contemporáneos: "a partir de ahora los filósofos de la política tienen que trabajar dentro de la teoría de Rawls o explicar por qué no lo hacen" (Nozick, 2015, p. 183), ${ }^{4}$ hasta nuestros maestros: "cualquier reflexión actual sobre justicia no puede menos de partir de su poderosa obra" (Villoro, 2007, p. 64). Sin embargo, hubo posturas más apacibles, en donde Rawls solo fue un elemento explicativo del cambio al interior de las ciencias sociales mas no una piedra angular de la filosofía política.

De lo dicho hasta aquí se desprende que en este contexto intelectual el retomo de la gran teoría en el ámbito de la filosofía moral y política vendría marcada por una vuelta a la tradición, es más a la tradición platónica, en la que Rawls tendría un lugar destacado, su contribución ha de verse en relación con las transformaciones en ese contexto más amplio de la reestructuración de las ciencias humanas, pero en ningún momento se sostiene que haya un antes y un después de 1971, es decir, de Rawls (Agra, 2004, p. 79).

Pese a ello no se puede negar que Rawls es un punto de partida para las discusiones teóricas o prácticas que busquen una manera de solucionar los diversos problemas sociales relacionados con justicia. En Colombia por ejemplo se recibió a Rawls en tanto posibilitó una lectura distinta de justicia distributiva que permitió a su vez una reinterpretación del orden normativo constitucional (Cortés, 1999), y sin embargo, su paradigma jurídico también debe ser reinterpretado ya que no pasó el riguroso examen de la pregunta por la legitimidad (Mejía, 1997). Esto es, Rawls sirvió a Mejía como un elemento hermenéutico de la constitución del 91 y, al mismo tiempo, para criticarla; la constitución fue injusta porque se fundó en un acuerdo social de un par de minorías dominantes mas no en un

\footnotetext{
3 la violencia desfundamentó la política (Arendt, 1951; 1958; 1970), aunque hay quienes afirman lo contrario (Schmitt, 1999a; 1999b). El trabajo de Dora Elvira García indica que a pesar de la ambigüedad, la violencia es un elemento importante y, quizá, fundacional de la sociedad (García, 2004).

${ }^{4} \mathrm{Al}$ que también se le crítica el estar sesgado por el velo universalista en el que piensa. En 1997, Delfín lo criticó a él y a su postura: “Nozick está atrapado, como lo estamos todos, en una cultura académica, en su caso la norteamericana, hecha de énfasis y olvidos selectivos" (Grueso, 1997, p. 18).
} 
consenso de las mayorías. Con ello se puede afirmar que Rawls construyó una teoría rigurosa y legítima, capaz de agenciar una esfera social diferente (justa), y sin embargo, quizá no real y difícilmente concreta; este fue otro punto de partida de la elección de este autor.

No obstante, este informe explora críticamente los gérmenes de lo que se conoce como teoría contractual de la justicia, es decir, lo referente a los elementos de más ideales como son la posición original, el velo de ignorancia y los principios de justicia; lo extracontractual refiere elementos del orden no ideal o formal de la misma, por ejemplo, la desobediencia civil ${ }^{5}$. Así, el artículo reconstruye los sentidos iniciales de los conceptos presenten en la obra de nuestro autor antes que fueran situados sustantivamente en su obra cumbre.

El artículo revisa críticamente los siguientes documentos rawlsianos: "Justice as Fairness" (1958), "The Sense of Justice" (1963) y "Distributive Justice" (1973) este último, quizá, el de mayor eco en la tradición filosófica occidental. En ellos se encuentran las primeras formulaciones de los conceptos sustantivos de la justicia. Formulaciones que se van decantando hasta llegar, por ejemplo, en justicia como equidad, a la desestimación del criterio de utilidad y a la primera formulación de los principios de justicia; en sentido de justicia, se va a encontrar la problemática de la persona del sujeto moral y del cómo está obligado a cumplir con la normatividad dada por los principios de justicia; y en justicia distributiva, la problemática radica en vislumbrar los principios de justicia y el velo de ignorancia como elemento fundamental para la justicia.

\section{2. "Justicia como equidad"}

El artículo "Justicia como equidad" expone una de las primeras concepciones de justicia, o al menos la más cercana a lo que se conocerá en Una teoría. Según Rodilla, el texto es "germinal", ya que presenta una de las versiones más cercanas, aunque no tan definida, a la justicia como imparcialidad. Rawls ya había desestimado el principio de utilidad para justificar las acciones de los agentes de la esfera social, pero también, terminó desestimándolo aunque en este ensayo lo examinó en tanto viable para las instituciones de la misma esfera.

El criterio de utilidad es inapropiado para seleccionar las razones que podemos esgrimir para justificar instituciones, porque enfoca el problema del correcto diseño de las mismas como un asunto de eficiente administración

\footnotetext{
Se sugiere consultar a Agra, En torno a la justicia: las aportaciones de Aristóteles, el pensamiento español del XVI, J. S. Mill, la fenomenología y Rawls. (1999).

${ }^{6}$ En adelante se refiere como: Justicia como equidad

${ }^{7}$ En adelantese refiere como: El sentido de justicia

${ }^{8}$ En adelante se refiere como: Justicia distributiva
} 
y asignación de recursos antes que con un problema de correcta definición de las relaciones intersubjetivas entre personas que, como partícipes en una actividad común, presentan pretensiones rivales (Rawls, 2012, p. 50).

También, cabe destacar que la justicia como equidad no obedece a la argumentación contractualista, el mismo Rawls señala que la teoría de los juegos es la más apropiada herramienta para solucionar los conflictos concernientes de ella. Mirémosle: la justicia como "equidad" [fairness], virtud de las instituciones sociales, entendida en tanto "debido trato entre personas que están cooperando o compitiendo unas con otras, como cuando se habla de juegos equitativos (fair games), competencia leal (fair competition) y negociaciones honestas (fair bargains)" (Rawls, 2012, p. 90), es expresada de manera determinante en el concepto pacto: "la justicia se piensa como un pacto entre egoístas racionales, cuya estabilidad depende de un equilibrio de poder y de la similitud de las circunstancias" (1958, p. 18). Una rigurosa búsqueda rawlsiana, rastrea en la tradición filosófica occidental tal concepto, lo encuentra en Platón, Epicuro y Hobbes. Esta justicia señala que el egoísmo en las personas e instituciones sociales implica la reciprocidad del mismo y la satisfacción de los intereses propios por encima de todos; tal egoísmo se acompaña de racionalidad, es decir, las personas e instituciones "conocen sus intereses propios con mayor o menor precisión; son capaces de adherirse a un curso de acción, una vez que han resuelto seguirlo " (13). Sin embargo, el "pacto" no posee ni la rigurosidad ni la universalidad que el contractualismo ofrece.

A cambio del universalismo Rawls obtiene de la teoría de juegos, indispensable en este desarrollo de justicia, la posibilidad de medir las condiciones de los escenarios de justicia, es decir, los aclara. ¿Qué es la teoría de juegos? Es una teoría o una estrategia formal que muestra de manera desencarnada el cómo y el qué de los conflictos morales, para allí optar por la mejor solución posible. No importa el virtuosismo o el egoísmo que se encarne en la acción de la persona o institución, la expone de manera formal, la desvela. El optar por éste o aquel mecanismo le compete a la teoría política dominante o, en el caso rawlsiano, a los principios de justicia. La teoría de juegos devela de manera precisa las complejas interacciones entre los agentes de la esfera social, es decir, gracias a esta teoría hay mayor posibilidad de que la ética consiga aplicar adecuadamente los principios de justicia. Así, la ética, que ha entrado en el discurso público, ${ }^{9}$ ha conseguido una herramienta valiosa en la búsqueda de mejores (justas) decisiones. La relación entre la teoría de los juegos y la justicia en el Rawls del 58 se estrecha ${ }^{10}$ en la medida que la primera posibilita un análisis riguroso de las condiciones bajo las cuales se aplican los principios de justicia: "su propósito reside, simplemente, en incorporar a la concepción de justicia las relaciones recíprocas entre los hombres

\footnotetext{
${ }_{9}^{9}$ Se sugiere consultar a Camps, El malestar de la vida pública (1996).

${ }^{10}$ Hasta el punto de considerar la teoría de juegos no como una simple herramienta útil, sino a la ética misma como una teoría de juegos. Se sugiere consultar a Harsanyi, Games and decision theoretic models in ethics (1992).
} 
que montan la escena para el planteo de cuestiones de justicia" (1958, p. 20). Sin embargo, Rawls no tarda en tomar distancia de ella dado que los agentes sociales no deciden sobre prácticas sino sobre criterios para establecer acciones justas:

[las partes, los agentes sociales] tampoco deciden, como en la teoría de los juegos acerca de las estrategias individuales ajustadas a las circunstancias en que respectivamente se encuentran en el juego... las partes acuerdan pautas de juicio, no una práctica determinada; no llevan a cabo ningún acuerdo o negociación específicos, ni estrategias particular alguna (2012, p. 88).

La intención de esta distancia le permite preparar el camino para acoger el constructivismo y sus elementos, como es el velo de la ignorancia y la posición original, mismos que no aparecen en la exposición de "Justicia como equidad".

De otra parte, los principios de justicia también aparecen, aunque no bajo la misma denominación que lo hace en Una teoría.

Primero, cada persona que participa en una práctica, o que se ve afectada por ella, tiene un igual derecho a la más amplia libertad compatible con una similar para todos; y segundo, las desigualdades son arbitrarias a no ser que pueda razonablemente esperarse que redundarán en provecho de todos, y siempre que las posiciones y cargos a los que están adscritas, o desde los que pueden conseguirse, sean accesible a todos (Rawls, 2012, p. 79).

Estos principios no obedecen al acuerdo hipotético de personas libres e iguales, como se desarrollan en 1971, sino a "una familia de principios que normalmente van asociados al concepto de justicia" (79). La exposición tiene un fuerte carácter inductivo, es decir, se parte de ellos para poder identificar el tipo de justicia necesaria para la legitimación de una esfera social, mientras que en 1971, ellos son introducidos como elementos originarios del experimento mental propuesto por Rawls, es decir, después de la consideración de la posición original. Estos principios de justicia son gérmenes de la teoría de la justicia, no desarrollan los principios en plenitud, sino, el primero, la igual libertad entre los agentes y, el segundo, las desigualdades que podrían ser justificadas en aras a la construcción de una esfera social justa.

En fin, se puede considerar que la justicia en este primer momento es introductoria, señala las vías que pretende desarrollar Rawls, ya sean los principios de justicia, o los criterios originarios que significan la justicia, ya el carácter constructivista, es decir, los argumentos que comienza a posicionar en contra del utilitarismo. Pero lo significativo de este intento, con especial consideración en la teoría de los juegos, es el esfuerzo de Rawls por no desencarnar la esfera pública, es decir, estructura una filosofía política en el que se exige el reconocimiento de sí mismo y de los otros como personas. 
Reconocer esos deberes en alguna medida, y con ello tener los elementos de la moralidad, no es asunto de elección, ni de intuir cualidades morales, ni de la expresión de sentimientos o actitudes (las tres interpretaciones entre las que frecuentemente oscila la opinión filosófica); es simplemente la posesión de una de las formas de conducta en las que se manifiesta el reconocimiento de los otros como personas (93).

Finalmente, cabe destacar dos problemas del texto en su exposición: 1) las desigualdades que podrían ser aceptadas en el segundo principio no explican suficientemente el beneficio que traerán para todos los agentes de la esfera social. 2) la exposición de la relación entre los dos principios y los conflictos contextuales originados en ella no explícita una solución para estos. Sin embargo, cabe acentuar el carácter inicial del texto, por lo que se necesitará de otros escritos rawlsianos que allanarán el camino para Una teoría.

\section{3. "Sentido de justicia"}

En el artículo "Sentido de justicia" se señalan los desarrollos de la moralidad con respecto a la internalización psicológica de los principios de justicia, es decir, el cómo un agente de la esfera social está en condiciones de acatar las acciones procuradas por los principios de justicia dados. Tales principios son los ya descritos en "Justicia como equidad", no hay diferencia en ellos, aquí lo que se amplía es la comprensión rawlsiana del sentido de justicia (entendido éste, como la posibilidad personal de diferenciar entre la bondad y la maldad, de sentir afecto y resentimiento, etc.; sin este sentido la esfera social carecería de algo esencialmente humano). En otras palabras, si Justicia como equidad expone los escondrijos analíticos de la justicia, sus principios y su aplicación por las instituciones sociales, "Sentido de justicia" valora y discute el cómo las personas cumplen con los principios. "La argumentación analítica no muestra que personas racionales, en tanto que participantes en un esquema de cooperación, vayan a hacer en casos concretos lo que la justicia exige" (Rawls, 2012, p. 106). Rawls recupera los estudios de psicología infantil hechos por Piaget: rescata los estadios en que el niño forma su criterio moral. En Piaget son la etapa pre-moral, la heterónoma y la autónoma, mientras que en Rawls "culpabilidad respecto de autoridades (authority guilt), culpabilidad respecto de asociaciones (association guilt) y culpabilidad respecto de principios (principle guilt)" (107). Antes de explicar los estadios del desarrollo moral rawlsiano, cabe destacar que la culpabilidad es el sentimiento moral preponderante, ¿por qué?, porque ella es un sentimiento moral que obedece inapelablemente a las actitudes naturales primitivas eminentemente humanas o actitudes de socialidad humana, es decir, en tanto que siempre está en relación a otros. La culpa está asociada en tanto sentimiento originario ${ }^{11}$

\footnotetext{
${ }^{11}$ Se sugiere consultar a Sartre, 2005. Un bosquejo de la teoría de las emociones.
} 
Un sentimiento moral según Rawls refiere en primera medida un concepto moral y los principios asociados a él, es decir, la carga de bondad o maldad (y la explicación racional y razonable de la misma) asociada a una acción serán determinante al levantar un juicio moral. La culpa es un sentimiento originario de lo humano que, en tanto sentimiento moral, enarbola la posibilidad de juicio moral. Así, el juicio desde la culpabilidad respecto de autoridad y de la asociación erigen a cualquier persona particular en tanto su objetivo: en la primera son los padres, en la segunda son los pares. El amor hacia los padres viene acompañado de autoridad que exige reparación cuando es trasgredida, la culpabilidad se hace presente en el transgresor; la relación entre pares viene acompañada por amistad y confianza en que cada uno haga lo que le corresponde, cuando no se satisface esta creencia las interacciones en la esfera social se ve afectada. Por su parte, el tercero no presupone ninguna persona o al menos una cercana, sino criterios de acción que deberán ser respetados en beneficio de la esfera social.

Sin embargo, a este desarrollo del sentido de justicia, se podría erigir una crítica que Villoro (2007) ha denominado la antinomia del sujeto moral. Esta se da porque se pretende estudiar a individuos abstractos en tanto su relación con el sistema general de normas, es decir, se escoge entre un sujeto autónomo capaz de legislar universalmente o un sujeto regido por el sistema de reglas sociales. Tal antimonia se puede evitar al partir de un individuo real. "Así, la antinomia se supera en acciones concretas. Es en la acción racional, personal o colectiva, donde se resuelve la antinomia planteada por la teoría" (2007, p. 107). Villoro, gracias a la psicología empírica, señala que no necesariamente existe una oposición entre el sujeto concreto y el sujeto generalizado. Villoro se apoyó en los estudios de Kohlberg; ${ }^{12}$ quien refiere el desarrollo moral desde un sujeto real. La persona real se relaciona con la norma de acuerdo a su proceso vital, cada vez tiene una comprensión más detallada y profunda de ella, lo que le permite tener una relación evolutiva con ella. Es en la última etapa de tales estadios, en donde la persona real puede decidir y actuar conforme a principios posconvencionales, universalmente válidos. La persona real es quien se rige por estas reglas, ella posee una comprensión distinta de la norma que le permite relacionarse de manera diferente con ella. En fin, el individuo del nivel posconvencial de Kohlberg es, al mismo tiempo, concreto y generalizado.

\footnotetext{
${ }^{12}$ La propuesta de Kohlberg tiene tres niveles con dos estadio cada uno: 1) el nivel preconvencional, refiere el seguimiento de las normas mediante el esquema clásico conductual, a saber, el estímulo-respuesta. Sus estadios son: a) obediencia y miedo al castigo y b) favorecer los intereses propios. 2) el nivel convencional, refiere que el seguimiento de las normas para favorezcan la aceptación por parte de los otros. Sus estadios son: c) expectativas interpersonales y d) normas sociales establecidas. 3) el nivel posconvencional, refiere el seguimiento de las normas mediante la internalización de los principios morales que las fundan. Sus estadios son: e) derechos prioritarios y contracto social y f) principios éticos universales (Kohlberg, 1992).
} 
Finalmente se puede aseverar que el sentido de justicia revisa las posibilidades reales de aplicación de los principios de justicia o al menos del cómo las personas pueden obligarse a ellos en situaciones concretas y particulares. Dada la exposición del carácter moral de la persona se puede aseverar junto a Rawls: "la capacidad para un sentido de la justicia es, pues, necesaria y suficiente para que el deber de la justicia se deba a una persona" (2012, p. 121). Sin embargo, parece que tal premisa no tiene cabida en la realidad social que enarbola el orden normativo; toca esperar a teoría de la justicia para validar la fuerza de esta premisa.

\section{Justicia distributiva}

El artículo Justicia distributiva hace referencia a los elementos propios de lo que se desarrollará en 1971; es un giro definitivo hacia Una teoría. La problemática radica en vislumbrar los principios de justicia en primera instancia. Pero también se erige el velo de ignorancia como elemento fundamental para la justicia. "Un velo de ignorancia evita que nadie resulte beneficiado o perjudicado por las contingencia de clase social y de buena o mala fortuna; de ahí que los problemas de negociación que surgen en la vida diaria a causa de la posesión de ese conocimiento no afecten a la elección de los principios" (Rawls, 2012, p. 125).

La discusión de los principios se introduce en la disputa con el utilitarismo; la que queda desestimada porque "le es indiferente, salvo de forma indirecta, la suma de satisfacciones entre individuos; igual que le es indiferente, salvo de forma indirecta, cómo se distribuye una persona sus satisfacciones a lo largo del tiempo" (124). Los argumentos a favor del contractualismo ya los hemos venido exponiendo, por lo que su elección no tiene contratiempos: "la alternativa más natural al principio de utilidad es su rival tradicional, la teoría del contrato social. El propósito de la doctrina contractualista es precisamente dar cuenta del carácter estricto de la justicia" (124). A Rawls le conciernen tres puntos de los principios:

Primera, cómo interpretar esos principios de modo que definan una concepción de la justicia completa y consistente; segunda, si es posible articular las instituciones de una democracia constitucional de modo que los dos principios queden satisfechos, al menos de forma aproximada; $y$ tercera, si la concepción de cuotas distributivas definida por ellos es compatible con nociones de sentido común (126).

El primer punto, en el que se pregunta por una correcta interpretación de los principios, se desarrolla en la discusión del principio de eficacia en la versión de Hume y desde el criterio de Pareto. El primero refiere la posibilidad de una interpretación personal adecuada, dado que un punto de referencia cualquiera puede mostrarse peor; el segundo se aplica a las instituciones y refiere la 
posibilidad de eficiencia cuando la distribución corresponde beneficiosamente a todos sin detrimento de alguno. De ambas se deduce la imposibilidad de una rigurosa interpretación tanto en el sentido común como en el formalismo de una teoría de la justicia; ambas configuran espacios de injusticia, el primero en el desconocimiento de la satisfacción personal, el segundo en el aseguramiento de justicia para todos los escenarios posibles. Así, se hace necesario una vía de interpretación rigurosa, que asegure la aplicación de la justicia tanto a nivel personal como institucional: ésta apela a una reinterpretación del segundo principio. Rawls acentúa la necesidad de un agente representante en el que se configure una distribución justa: el menos favorecido. De esta manera, aparece la formulación del segundo principio:

Dando por supuesto que se da el marco institucional requerido por la igualdad equitativa de oportunidades, las mayores expectativas de los que están mejor situados en la estructura básica son justas si y solo si operan como parte de un esquema que mejora las expectativas de los miembros menos favorecidos de la sociedad (137).

Este nuevo elemento, el menos favorecido, permite evaluar la coherencia de los principios frente a las instituciones democráticas; se emprende un trabajo analítico riguroso para conceptualizarlos frente a las máximas expresiones de la democracia: el grito de la revolución francesa y el imperativo categórico en su segunda expresión: "Obra de tal modo que uses la humanidad, tanto en tu persona como en la de cualquier otro, siempre como un fin, y nunca sólo como un medio" (Kant, 2007, p. 44). Frente al primero acentúa el principio de la fraternidad con la segunda parte del segundo principio; mientras que a la libertad y la igualdad con el primer principio y la primera parte del segundo, respectivamente. La fraternidad no puede ser un sentimiento irreal de lazos sociales o personales, sino que, unido al principio de la diferencia, "corresponde a un significado natural de la fraternidad: a saber, a la idea de no desear tener mayores ventajas salvo que sea en beneficio de los que están peor" (140). Si el principio de fraternidad que, considerado el de menor importancia por la tradición política, se afianza a la luz de los principios de justicia, se puede señalar la valía de los demás principios de la revolución: a saber, la libertad en tanto posible en su esquema más extenso, y la igualdad en cuanto a la "equitativa igualdad de oportunidades". Mientras tanto, respecto del imperativo categórico, afirma que el principio de utilidad es inaplicable en las instituciones democráticas porque permite tratar a las personas al mismo tiempo como fines y como medios, por lo tanto, afirma que los principios de justicia no tratan a las personas bajo ninguna circunstancia como medio; el problema en este sentido es que no destaca la manera en que los principios valoran a las personas como fines. Cabe destacar que en este texto no lo aclara del todo y toca esperar a 1971 en donde si explicita en qué sentido el imperativo categórico refiere a las personas tan sólo como fines. Así, bajo el rigor y el beneplácito de estas expresiones democráticas se puede asegurar con mayor medida la plausibilidad 
de los principios de justicia. El segundo, el de la adecuación de los dos principios de justicia para con las instituciones representativas de una democracia, esboza el siguiente argumento:

Si la ley y el gobierno actúan de forma efectiva para mantener mercados competitivos, pleno empleo de recursos, amplia distribución de la propiedad y la riqueza a lo largo del tiempo, y para mantener el mínimo social apropiado, entonces, si existe igualdad de oportunidades adecuada mediante un derecho de todos a la educación, la distribución resultante es justa (145).

Hasta aquí no hay diferencia alguna con otras argumentaciones y/o utopías sociales, pero Rawls ha introducido el principio de diferencia: quien tendrá la misión de buscar la fundamentación de lo pretendido en este numeral; quizá sea esto lo más provocativo de la arqueología de la teoría de la justicia presentada hasta aquí por Rawls: en otras palabras la preocupación no resuelta, pero sí explícita es el cómo aplicar los principios de justicia evitando la pérdida de eficiencia. Por su parte, el tercer problema esboza la conclusión del documento, que no tiene otra intención que la de discutir desde lo entendido por recto como justicia social en tanto le corresponde la "distribución igualitaria" y el "bienestar total", esto es, maximizar la suma de expectativas sujetándose a la restricción de que no se permite que nadie caiga por debajo de un cierto nivel de vida reconocido" (155).

\section{Conclusiones}

El material presentado esboza al menos tres tesis y/o momentos que hubieran superado en parte las antítesis que surgieron para 1971. Otro de los beneficios de la revisión de los textos fue el reconocimiento y el posicionamiento temprano de las categorías de Una teoría. Es decir, para 1971, elementos como la posición original, el velo de la ignorancia y los propios principios de la justicia, ya habían tenido discusiones importantes. El segundo logro de esta indagación está en la "arqueología" hecha, es decir, en la condición metodológica para la investigación emprendida. Revisar la argumentación y construcción es fundamental para el posicionamiento de la teoría de la justicia, al menos, siguiendo a Agra, uno "aterrizado". Rawls no es una figura menor en la filosofía, aunque tampoco es un imprescindible. Esta indagación y las páginas que faltan, lo tomará de manera mediana, es decir, Rawls es una figura temprana para la filosofía política.

Rawls abre el paradigma de la justicia social en pro de los menos aventajados. El contractualismo también se abre espacio en estos primeros textos. Pero el punto fundante aparece en la justificación que hace de la posición original. En ella pasa de la teoría de los juegos a la idealidad de los contractualistas, o al menos prepara el terreno para ésta. La teoría de los juegos se inserta en tanto estrategia para definir soluciones a los problemas de la sociedad $y$, sin embargo, no es suficiente 
para establecer sujetos morales libres e iguales. Allí radica la diferencia y, también, la ganancia de esta revisión, ya que se indica que los problemas de la sociedad requieren un posicionamiento distinto de los agentes sociales. El velo de la ignorancia es el elemento de la teoría ideal rawlsiana que consigue este paso y abre la discusión por la justicia.

La ganancia final es para la investigación en general, a saber, una revisión completa de Rawls. Los pretextos de la justicia rawlsiana abren la discusión por el rol de Una teoría en la historia de la filosofía y también la cierran. El discusión por la justicia social no se abrió en 1971, tampoco se cerró allí. Es una discusión que aún tiene labores pendientes que deben situarse en posicionamientos políticos y filosóficos coherentes y rigurosos $\varphi$

\section{REFERENCIAS}

Agra, M. X. (1999). En torno a la justicia: las aportaciones de Aristóteles, el pensamiento español del XVI, J. S. Mill, la fenomenología y Rawls. Madrid: Eris.

Agra, M. X. (2004). "Antes y después de Rawls". Revista internacional de filosofía política, (23), 71-92.

Arendt, H. (1993). La condición humana. Barcelona: Paidós.

Arendt, H. (2006). Los orígenes del totalitarismo. Madrid: Alianza.

Arendt, H. (2006). Sobre la violencia. Madrid, Alianza.

Camps, V. (1996). El malestar de la vida pública. Barcelona: Grijalbo.

Cortés, F. (1999). De la política de la libertad a la política de la igualdad: un ensayo sobre los límites del liberalismo. Bogotá: Siglo el hombre.

García, D. E. (2004). La violencia como condición o como disolución de lo político: Carl Schmitt y Hannah Arendt. Signos Filosóficos, VI (11), 73-96.

Grueso, I. (1997). Rawls. Una hermenéutica pragmática. Cali: Universidad del Valle.

Harsanyi, J. C. (1992). Games and decision theoretic models in ethics. Paises Bajos: Norht Hollan.

Kant, I. (2007). Fundamentación de la metafísica de las costumbres. San Juan de Puerto Rico: Pedro Rosario Barbosa. 
Kohlberg, L. (1992). Psicología del desarrollo moral. Bilbao: Desclée de Brouwer.

Mejía, O. (1997). Justicia y democracia consensual. Bogotá: Ediciones Uniandes y Siglo del Hombre.

Nozick, R. (2015). Anarquía, estado y utopía. Madrid: Innisfree.

Rawls, J. (1958). "Justice as Fairness". The Philosophical Review. LXVII 164-194. Ithaca, New York.

Rawls, J. (1963). "The Sense of Justice". The Philosophical Review. LXVII. 281-305 Ithaca, New York.

Rawls, J. (1973). “Distributive Justice". Phelps. Economic Justice. Londres: Penguin Books.

Rawls, J. (1995). Una teoría de la Justicia. México: Fondo de Cultura Económica.

Rawls, J. (2012). Justicia como equidad. Materiales para una teoría de la justicia. Madrid: Técnos.

Sartre, J. P. (2005). Un bosquejo de la teoría de las emociones. Madrid: Alianza.

Schmitt, C. (1999a). El concepto de lo político. Madrid: Alianza.

Schmitt, C. (1999b). La dictadura. Madrid: Alianza.

Villoro, L. (2007). Los retos de la sociedad por venir. Ensayos sobre justicia, democracia y multiculturalismo. México: Fondo de Cultura Económica. 